\title{
Protée
}

\section{Aloysius Bertrand : la volonté de transposition}

\section{Luc Bonenfant}

Volume 31, numéro 1, printemps 2003

\section{La transposition générique}

URI : https://id.erudit.org/iderudit/008499ar

DOI : https://doi.org/10.7202/008499ar

Aller au sommaire du numéro

Éditeur(s)

Département des arts et lettres - Université du Québec à Chicoutimi

ISSN

0300-3523 (imprimé)

1708-2307 (numérique)

Découvrir la revue

Citer cet article

Bonenfant, L. (2003). Aloysius Bertrand : la volonté de transposition. Protée, 31(1), 27-36. https://doi.org/10.7202/008499ar

\section{Résumé de l'article}

Cet article étudie les mécanismes de transposition dans Gaspard de la Nuit. Convoquant tout à la fois des codes endogènes et exogènes au système de la littérature, les poèmes en prose de ce recueil signalent une différence entre la " transposition générique " et la « transposition modale " : si la première concerne l'aspect plus proprement formel des textes, la seconde en transforme plutôt les visées, les desseins. Comme opération de combinaison, la transposition dépend donc de la distinction entre le genre et le mode. Enfin, cette distinction permet aussi de souligner le caractère résolument actif de l'opération de transposition, qui se distingue ainsi des processus de différenciation et d'hybridation. 


\section{ALOYSIUS BERTRAND: LA VOLONTÉ DE TRANSPOSITION}

LUC BONENFANT

Du fait qu'il allie la poésie à la prose, le genre du poème en prose semble remettre en cause les fondements de la traditionnelle division des genres en lyrique, épique et dramatique ${ }^{1}$. Le genre se trouve toutefois aussi informé par des codes artistiques exogènes, tels que la peinture et la musique. Parmi plusieurs, David Scott (1989) a étudié la structure spatiale du genre, et si l'influence de la musique chez les poètes en prose a moins retenu l'attention des spécialistes, on a toutefois noté que le genre inspire, souvent de façon importante, des compositeurs modernes: Maurice Ravel met en musique trois poèmes d'Aloysius Bertrand, alors que Benjamin Britten adapte les Illuminations d'Arthur Rimbaud.

Sur le plan de son organisation, le poème en prose possède donc des particularités qu'il emprunte, mais non exclusivement, au vers, à la peinture, à la musique et aux genres brefs. Il convoque plusieurs poétiques qui, pour autant qu'elles soient différentes, n'en demeurent pas moins compatibles. Ces poétiques diverses concernent tout autant le genre que le mode, «invit[ant] enfin le lecteur à se demander ce qui fait qu'un texte est ou non reçu comme poème» (Sandras, 1995: 13).

Ainsi, le poème en prose apparaît comme un terrain privilégié pour explorer les différentes facettes de la notion de transposition générique, qu'on définira comme «la reprise de traits génériques caractéristiques d'un genre donné dans des œuvres où ils semblent plus inattendus" (Dion, Fortier et Haghebaert, 2001: 353-354)2. Partant de cette définition, et puisant des exemples dans l'œuvre d'Aloysius Bertrand, nous montrerons d'abord que la transposition n'est pleinement opératoire qu'une fois envisagée sur le double plan du genre et du mode. Cette distinction nous permettra ensuite de souligner le caractère résolument actif, voire engagé, de l'opération de transposition, qui se distingue par là des processus de différenciation et d'hybridation ${ }^{3}$.

\section{LA TRANSPOSITION GÉNÉRIQUE}

Plusieurs des poèmes contenus dans Gaspard de la Nuit sont lisibles en tant que récits: ils mettent en place une diégèse, un narrateur, des personnages, etc., ce qui n'est au demeurant guère surprenant: 
[...] la narration et l'allure du conte s'associent assez bien aux structures poétiques. Le poème en prose, parce qu'il revêt souvent un habillage narratif, côtoie le conte ou la nouvelle et ceux-ci, inversement, peuvent facilement prendre une dimension poétique. (Vincent-Munnia, 1996: 224-225)

En conséquence, Bertrand adapte des procédés narratifs dans les textes de son recueil. «La viole de Gamba» (2000: 127-128), dont l'argument fantaisiste se trouve réparti selon trois courts épisodes ${ }^{4}$, utilise les formules narratives de la commedia dell'arte telles que la reprise d'un canevas simpliste et la mise en scène de personnages qui sont autant de parangons (Pierrot, Colombine, Arlequin). «La poterne du Louvre» raconte plutôt l'histoire d'un nain qui effraie le garde du Louvre en lui faisant croire qu'il est le diable. Comme dans plusieurs des poèmes de Gaspard de la Nuit, cette narration est exposée par un narrateur extradiégétique.

Travaillant avec un matériau jusqu'alors principalement utilisé pour raconter des histoires - la prose -, Bertrand n'hésite pas à recourir aux ressources des genres narratifs, qu'il transpose adéquatement dans les textes de Gaspard de la Nuit. Cependant, il ne limite pas son utilisation de la prose à la reprise de procédés narratifs; dans son recueil, l'auteur n'hésite pas à transposer aussi des procédés qu'il emprunte au vers. Parmi ceux-ci, la division en couplets et la récurrence anaphorique. «Harlem» (2000: 115-116) est exemplaire de ces deux emprunts bertrandiens au vers. D'abord, chaque strophe du poème est séparée des autres par un blanc typographique. Ces blancs interstrophiques agissent sur le rythme même de la lecture du texte puisqu'ils commandent une pause de la lecture semblable à celle imposée par le blanc qui suit traditionnellement le vers du poème. Ces pauses de la lecture, Bertrand semble bien les avoir voulues: «il jettera de larges blancs entre ces couplets comme si c'étaient des strophes en vers" (2000: 373), écrit-il dans ses directives typographiques. La succession des strophes et des blancs lui permet de faire passer délibérément dans le domaine de la prose un procédé jusque-là réservé au vers.
La prose, normalement, emplit la page, sur sa largeur et dans sa hauteur; le blanc au contraire est traditionnellement l'apanage $d u$ vers qui, par ses retours réguliers à la ligne et ses regroupements en strophes, crée du vide et le vide autour de lui (ainsi que du silence, un rythme particulier, des marges de rêverie...). (Vincent-Munnia, 1996: 106)

Tous les poèmes de Gaspard de la Nuit utilisent ce procédé du blanc typographique ${ }^{5}$, que la critique a assez commenté pour qu'il soit inutile d'insister. Mieux vaut remarquer que Bertrand emprunte aussi au vers sa métrique, pour mieux l'adapter aux besoins de la prose. Les strophes de «Harlem» sont par exemple toutes d'une longueur de trois lignes; elles reprennent ainsi le compte du pied - lequel appartient historiquement au vers - pour le remplacer par un compte plus approprié à la prose: la ligne (Bonenfant, 2002: 120). À première vue, la répétition de la conjonction «et» à chaque début de strophe rappelle les récurrences anaphoriques qu'on trouve dans nombre de poèmes versifiés. Mais elle appelle aussi le principe de liaison de coordination propre au vers. Du fait qu'il s'écrit sur une seule ligne, le vers implique inévitablement l'idée de «retour». Un vers n'est pas une unité sémantique et syntaxique isolée; il se lit toujours dans un rapport de coordination avec les autres vers du poème. Bertrand adapte donc dans son poème un autre de ses procédés: il remplace l'idée de retour par celle de la récurrence anaphorique, plus juste en regard de la nature du matériau d'écriture avec lequel il travaille.

La répétition de «Il s'assied» dans «Le capitaine Lazare», du «Écoute! - Écoute!» dans «Ondine»; la reprise du "ainsi j'ai vu, ainsi je raconte» d' "Un rêve» et le quadruple «Notre-Dame d'Atocha, protégez-nous! s'écriaient les brunes andalouses, nonchalamment bercées au pas de leurs mules» dans «Les muletiers" ne sont que quelques-unes des nombreuses récurrences anaphoriques présentes dans Gaspard de la Nuit, ce qui permet à Gisèle Vanhèse Cicchetti d'écrire:

Quand le lecteur parcourt ces poèmes en prose qui, dans

Gaspard, s'articulent en strophes de longueur presque égale, aux constructions symétriques bien marquées et dans certains cas 
suivies d'un refrain, il perçoit leur hétérogénéité par rapport à l'esthétique de son temps et, s'il dispose d'une bonne culture littéraire, il la relie à un type de poésie du passé, elle aussi à forme fixe: la ballade. (1979: 12)

Ainsi, la transposition générique propre aux poèmes en prose bertrandiens affecte d'abord la structure des textes, lesquels empruntent des procédés formels au vers et aux genres narratifs brefs pour se constituer comme poèmes en prose. Ces transpositions génériques indiquent de façon exemplaire que le poème en prose est justement cela: un poème en prose, c'est-à-dire un poème qui, partant d'une tradition précise et de ses techniques (celles du vers), se sert plutôt des ressources propres à la prose pour s'élaborer en poème. Alastair Fowler a raison d'écrire que

[...] far from inhibiting the author, genres are a positive support.

They offer room, as one might say, for him to write in - a habitation of mediated definiteness; a proportioned mental space; a literary matrix by which to order his experience during composition. (1982: 31)

Lorsqu'il s'attache à la composition de Gaspard de la Nuit vers 1828, Aloysius Bertrand a déjà écrit de nombreux vers. Il a donné d'audacieux poèmes, dont des dizains en losange et des études de vers impairs. Il a aussi écrit d'excellents sonnets, dont le fameux « $\grave{A}$ monsieur Eugène Renduel» (2000: 522). Bertrand maitrise bien les techniques du vers; toutefois, il ne se contente pas de simplement les reprendre dans ses poèmes en prose. Plutôt, il adapte ces techniques au nouveau matériau du poème qu'est la prose. La reprise bertrandienne des techniques du vers n'est pas qu'une répétition; elle devient véritablement une transposition, du fait même de son adaptation aux besoins du nouveau genre, montrant par là la part active du génie de son auteur ${ }^{6}$.

En véritable écrivain qu'il est, Bertrand ne se préoccupe pas des seules techniques scripturaires. Par exemple, la transposition du blanc typographique décrite plus haut n'agit pas sur le seul plan formel des textes, mais aussi sur le plan de leur situation d'énonciation. À propos des deux versions de «La citadelle de Wolgast», Max Milner écrit:

[...] dans la version définitive, ne subsistent plus que quelques détails, séparés par un blanc qui résume mieux que toute parole le tragique de la situation et l'isolement de la citadelle héroïque.

(1980: 27)

Bien qu'inspiré d'une technique appartenant au vers, le blanc bertrandien laisse rapidement transparaitre une attitude d'ordre modal plutôt que strictement générique. Cette reprise du blanc indique que l'auteur attache tout autant d'importance à circonscrire le dessein de ses textes qu'il a porté attention à leur composition formelle. Dans la lettre citée à son typographe, il demande de «blanchir comme si le texte était de la poésie» (2000: 373). L'utilisation de la conjonction indique certes trop bien l'ambivalence de Bertrand, qui ne sait trop comment classer ses textes ${ }^{7}$, mais, du point de vue qui est ici le nôtre, cette conjonction manifeste aussi une prescription certaine quant à la façon dont il faut lire ces textes. Bien avant le genre, l'intention auctoriale déclarée concerne le mode des textes, qui sera celui de la poésie. Elle laisse ainsi soupçonner que la transposition opérée par Bertrand dépasse les questions de technique liées aux genres pour déboucher plus largement sur le plan modal des textes ${ }^{8}$.

L'exemple de Gaspard de la Nuit indique que l'on doit opérer une distinction entre la transposition générique et la transposition modale, lesquelles jouent sur des plans différents de l'œuvre: celui de sa «forme compositionnelle» (laquelle dépend par ailleurs d'une série diverse de facteurs ${ }^{9}$ ), celui de sa «forme architectonique» (Bakhtine, 1978) ${ }^{10}$. Comme opération de combinaison, la transposition dépend nécessairement de la distinction genre/mode.

Alastair Fowler $(1974 ; 1982)$ a montré que les genres connaissent habituellement trois phases: 1 . celle de leur émergence; 2 . celle de leur constitution; 3 . celle de leur transformation. La troisième phase se constitue le plus souvent par la médiation genre/mode:

This restatement suggests a general hypothesis: namely that genre tends to mode. The genre, limited by its rigid structural 
carapace, eventually exhausts its evolutionary possibilities. But the equivalent mode, flexible, versatile, and susceptible to novel commixtures, may generate a compensating multitude of new generic forms. (1974: 92)

Dès lors, si la transposition générique révèle l'hybridation générique d'un texte, la transposition modale appelle plutôt la transformation historique du genre.

Parce qu'elle relève de l'invention formelle, la transposition générique laisse poindre le métissage compositionnel des textes. La présence simultanée, dans Gaspard de la Nuit, de caractéristiques propres au vers (le blanc typographique, la récurrence) et aux genres narratifs brefs (le narrateur, la transformation événementielle diégétique) en sont des exemples.

La transposition modale transforme quant à elle la visée d'un genre, elle permet de redéfinir le rapport particulier d'un genre à ses thèmes. En somme, elle permet à l'écrivain qui la pratique de modifier les balises d'un genre pour qu'il emprunte le dessein d'un autre de façon à parler différemment d'un sujet particulier. Dion, Fortier et Haghebaert parlent à cet égard de "changement de registre» (2001: 354). La performativité du poème en prose, empruntée au genre théâtral de façon à ce que le genre ressemble parfois à la saynète, en est un exemple ${ }^{11}$. De même,

[...] l'autobiographie africaine, qui tend à greffer à l'introspection individuelle caractéristique du genre en Occident un récit de l'expérience collective, constitue un exemple éloquent d'un tel décalage. (2001: 354)

Bertrand lui-même pratique ce type de transposition lorsqu'il crée les fantaisies novatrices de Gaspard de la Nuit.

\section{LA TRANSPOSITION MODALE}

S'inspirant de la critique génétique, les commentateurs s'entendent pour remarquer que

[...] la concentration formera une part importante de l'art

d'Aloysius Bertrand. Ce procédé, qui suggère par touches légères et rapides, par une suite d'évocations parfois brutales, réduit le décor à son expression la plus simple. (Corbat, 1975: 36)
Partant d'une première version clairement narrative, par moments prosaïque, Bertrand arrive à créer des textes poétiques. C'est ainsi qu'il emprunte à un mode ses procédés pour en créer un autre.

En témoignent «L'air magique de Jehan de Vitteaux" (2000: 271-272) et son avant-texte, «La gourde et le flageolet" (2000: 364). Ce dernier rappelle, par sa forme et sa visée, les récits brefs romantiques. D'abord, il renferme un argument narratif, qui se comprend selon l'ordre séquentiel et hypotaxique de sa diégèse ${ }^{12}$. Ensuite, il s'ouvre sur une formule qui rappelle le «Il était une fois» du conte ${ }^{13}$. De même, son narrateur met en évidence le caractère typé des personnages, qui deviennent autant de parangons qui s'opposent au sein de la trame narrative ${ }^{14}$. Enfin, le texte se termine sur une moralité dont la dernière proposition, «mais au diable si je bois jamais à la gourde d'un vilain!» (2000: 364), confère au texte une valeur narrative d'exemplum.

Bertrand reprend ces éléments dans «L'air magique de Jehan de Vitteaux", mais d'une manière fragmentaire, suggérant là que le second texte transfère sur le mode poétique les éléments narratifs de la première version. Par exemple, la première strophe présente les mêmes deux personnages, mais sur le mode de la juxtaposition plutôt que de l'enchaînement narratif:

La feuillée verte et touffue: un clerc du gai savoir qui voyage avec sa gourde et son rebec, et un chevalier armé d'une énorme épée à couper en deux la tour de Montlhéry. (2000: 271)

De même les deux sections de la phrase séparées par le deux-points se trouvent dans un rapport de simultanéité plutôt que de conséquence. Insécable, l'image rompt avec une éventuelle linéarité narrative. Aussi, la relative absence d'adjectifs qualificatifs au sein de la strophe montre que les descriptions y sont minimales; en utilisant principalement des noms communs, Bertrand semble suggérer qu'il faille laisser à l'imagination du lecteur le soin de visualiser la scène comme il l'entend. L'auteur procède par touches suggestives et lacunaires qui disent finalement beaucoup plus qu'elles ne paraissent, si ce n'est que 
parce qu'elles ne disent rien de l'état du clerc, de ses instruments, ou du chevalier.

Sans totalement supprimer le caractère anecdotique de son texte, Bertrand le transforme d'une manière telle que, du mode narratif, l'histoire racontée se trouve rapidement transposée sur le mode poétique. Bertrand utilise les ressources propres aux genres narratifs brefs, tels que l'usage du parangon, pour finalement livrer à ses lecteurs un texte que la postérité considérera comme un poème en prose, c'est-à-dire comme un texte en prose fonctionnant sur le mode de l'implicite et de la suggestion que l'on connaît à la poésie.

Pour des raisons notamment liées à la notion d'individualité caractéristique de l'ethos de l'époque, la poésie romantique se voit largement envahie par le mode lyrique, mode que Bertrand n'hésite pas à utiliser dans certains des textes de Gaspard de la Nuit. Dans «Encore un printemps», l'instance énonciatrice du poème se lamente sur les difficultés d'une vie trop pleinement sentie: «Ô ma jeunesse, tes joies ont été glacées par les baisers du temps, mais tes douleurs ont survécu au temps qu'elles ont étouffé sur leur sein». (2000: 245). S'adressant aux femmes de sa vie passée, le "Je" ne les considère pas comme de simples objets à décrire. Il se fusionne véritablement à elles; sa voix est un point de jonction à travers lequel il peut exprimer sa déception face aux tricheries de la vie. Ainsi,

[...] le "sujet lyrique» n'est pas le centre-source d'une parole qui

l'exprime, mais plutôt le point de tangence, l'horizon désiré

d'énoncés subjectifs ou non qu'il s'attache à relier [...].

(Rabaté, 1996: 67)

Fonctionnant plutôt sur le mode de la négation, l'énonciation lyrique de "À M. David, statuaire" permet au sujet de s'exprimer pleinement sur les malheurs relatifs à l'amour et à la gloire. Le poème chante les circonstances de l'affliction du sujet qui exprime, à la première personne, son malheur personnel: «et j'ai prié, et j'ai aimé et j'ai chanté, poète pauvre et souffrant!» (2000: 282). Le sujet du poème cherche à s'unir à une autre voix qui comprendra son trouble, et répondra (peut-être) par la voie/voix du réconfort: «Ah! l'homme, dis-le moi, si tu le sais, l'homme, frêle jouet gambadant suspendu aux fils des passions, ne serait-il qu'un pantin qu'use la vie, et que brise la mort?» (2000: 282). Le sujet lyrique du poème [...] se détermine ainsi non dans un rapport autocentrique à lui-même mais dans la relation qu'entretient sa propre voix avec celle d'une communauté humaine symbolisée par [l'autre].

(De Sermet, 1996: 86)

Avant Bertrand, d'autres auteurs avaient créé une prose lyrique. Rousseau et Chateaubriand ont laissé des proses qui se détachent du prosaïsme pour accéder à la poésie du rêve. Si cette prose lyrique peut être considérée poétique dans la mesure où elle laisse place à des épanchements sentimentaux, elle s'oppose toutefois au lyrisme, qui est le mode d'énonciation particulier d'un sujet en quête. Car le sujet lyrique «n'est donc pas à entendre comme un donné qui s'exprime selon un certain langage, la langue changée en chant, mais comme un procès, une quête d'identité" (Rabaté, 1996: 66) 15. L'innovation de Bertrand réside justement dans ce que l'emprunt des thèmes les plus courants de la prose lyrique (rêverie, épanchement, émotion) lui permet de faire passer vers la prose un mode d'énonciation particulier - le lyrisme -, jusque-là réservé au vers.

Puisant aux ressources de la littérature, Bertrand subvertit la narrativité traditionnelle de la prose et le lyrisme du vers de façon à créer des textes originaux du point de vue du mode. Une telle transposition modale est bien entendu endogène au système de la littérature, puisqu'elle convoque des codes puisés au sein des modes littéraires «traditionnels» que sont l'épique, le lyrique et le dramatique. Parallèlement à ce type de transposition, il existe aussi une transposition modale exogène au système de la littérature. Un auteur peut choisir de transposer sur le plan littéraire des codes qui appartiennent traditionnellement à d'autres arts. Les poèmes en prose de Gaspard de la Nuit fournissent deux exemples de ce type de transposition.

Si le blanc typographique du vers sert à Bertrand pour diviser formellement les strophes de ses poèmes, 
il lui permet aussi de transposer une intention de type modal: celle du cadre pictural. En effet, les poèmes bertrandiens «se présentent sous une forme fortement structurée" (Vincent-Munnia, 1996: 93); ils possèdent une nette "unité architecturale [dont] le plan, du reste, ne varie guère: entre un prologue et un épilogue, trois ou quatre couplets symétriquement distribués" (Bernard, 1959: 62). On peut penser qu'ils se présentent comme autant de tableaux qui doivent être lus dans l'unité cadrée de leur signification. Ces poèmes indiquent combien Bertrand a été sensible à la qualité unitaire des tableaux auxquels il fait référence dans le sous-titre de son recueil, et qu'il a vraisemblablement cherché à reproduire cette unité dans sa prose.

Cet intérêt pour la transposition picturale n'est bien entendu pas nouveau chez Bertrand. En 1828, l'auteur donnait trois compositions dans Le Provincial qu'il a appelé des bambochades ${ }^{16}$. Or la bambochade est un genre pictural qui met en scène le burlesque de la vie quotidienne, qu'on trouve aussi, mais sous une forme plus hallucinée et fantastique, chez Callot. Il semble bien que

Bertrand était donc, avant même de placer ses poèmes sous le patronage de Rembrandt et de Callot, à la recherche de certaines équivalences entre la poésie et la peinture, et cela pour évoquertoujours avec une pointe d'humour - des scènes "prises sur le vif", situées dans un ailleurs de convention. (Milner, 1980: 13)

La transposition des cadres picturaux dans le domaine de la poésie n'est pas que formelle. Bertrand ne se contente pas de simplement reproduire fidèlement sur la page les cadres de la peinture. Le cadrage des poèmes en prose bertrandiens sert à exacerber leur aspect poétique, dans la mesure où ils ne fonctionnent pas tant sur le mode de la litote que sur celui de l'indicible: muets, les cadres disent tout de même ce qui ne peut être explicité. La transposition du cadre pictural dans la prose $\mathrm{du}$ poème sert le transfert d'une vision du monde; elle s'inscrit donc modalement dans la poésie des poèmes. Par exemple, Francis Claudon a noté que Gaspard de la Nuit «paraît nettement paraphraser Rembrandt et la pente tortueuse de sa rêverie» (1988: 78). La combinatoire opérée par le transfert du cadre pictural dans le domaine littéraire permet de raffiner les modalités poétiques du poème, qui acquiert sa poéticité par l'accession au mode de la rêverie (Rembrandt) et du fantastique (Callot):

Ainsi la parenté entre les deux artistes [Bertrand et Callot] est-elle moins à chercher dans le choix des motifs qui excitent fortement l'imagination, que dans le jeu entre l'esprit spiritose, vivifié par l'humour, et l'esprit spirituale, celui du rêve et de la méditation. (Choré, 1993: 108)17

C'est donc là que la transposition, par Bertrand, du blanc pictural dans le domaine de la prose diffère de la reprise technique du blanc du vers: le premier concerne le mode des textes bertrandiens, le second regarde leur forme. Cette transposition modale, Bertrand l'opère toutefois aussi à partir de la musique, de laquelle il emprunte d'abord un nom (fantaisie), ensuite une notion (la liberté).

Avant Bertrand, Hoffmann avait procédé à une transposition littéraire de la musique. Les

Fantasiestücke in Callots Manier sont exemplaires du double parcours de l'Allemand, qui fut écrivain et compositeur ${ }^{18}$. Quelques années plus tard, et dans le contexte fort différent qu'est celui du champ littéraire français de l'époque, Bertrand s'inspire d'Hoffmann pour transposer dans le domaine de la littérature les possibilités formelles et modales d'un genre jusque-là musical: la fantaisie (Bonenfant, 2002: 247-252).

Dès son apparition au XVIe siècle, la fantaisie musicale se distingue par son invention formelle et son refus du conformisme. À l'époque romantique, le genre apparaît comme un phénomène arbitrairequi défie toutes les règles formelles:

[...] the attempt to bypass the artistic norms current at any time and, independently of them, to allow the creation of a musical work to depend exclusively on the inventive powers (fantasy) of the artist appears thus. (Schleuning, 1971:6)

En libérant ses poèmes du carcan du vers, Bertrand peut transposer en littérature cette idée de liberté propre à la fantaisie musicale. Ce faisant, il inscrit ses 
poèmes dans le cadre (modal) plus large qu'est celui de la fantaisie telle que l'entendent les romantiques. Les romantiques français associent la fantaisie à la fausseté de l'art, au sens où Platon entendait cette fausseté; elle est pour eux synonyme d'imagination et de caprice. Le choix de Bertrand de retenir le nom «fantaisie" pour ses poèmes lui permet de transposer l'idée de liberté du genre musical, laquelle correspond précisément à l'idée que ses contemporains se font de la poésie. La transposition formelle devient ainsi rapidement une transposition modale.

Le recueil de Bertrand montre bien que la reprise d'une caractéristique formelle telle que la division en couplets (que l'auteur prend du poème en vers) diffère de la mobilisation narrative du genre (empruntée aux genres brefs) ou encore de la convocation de la liberté musicale, justement parce qu'elle joue à un autre niveau de l'œuvre. En conséquence, il semble essentiel de distinguer entre la transposition générique laquelle concerne la reprise d'éléments plus strictement formels d'un genre - et la transposition modale, qui reprendra surtout un esprit, une visée.

Au plus fort de l'époque romantique, Bertrand pratique des genres courants tels que le poème en vers irréguliers, la nouvelle et le conte. Bertrand aurait pu choisir de poursuivre ses expérimentations sur le vers, et se satisfaire de perfectionner un art qu'il maîtrisait déjà assez bien. Il n'hésite pourtant pas à transposer dans le matériau de la prose des procédés techniques et modaux qui avaient jusqu'alors appartenu respectivement au vers et aux genres narratifs brefs, mais aussi à la peinture et à la musique. Ainsi, il ne se contente pas de simplement reproduire des techniques précises, de reprendre fidèlement un esprit ou une visée; plutôt, il les traduit pour les besoins de la prose (traducere $=$ faire passer). Ses transpositions sont de véritables adaptations, dans la mesure où les proses contenues dans Gaspard de la Nuit sont entièrement nouvelles, tout en reposant sur des traditions appartenant à d'autres arts.
La transposition bertrandienne des genres et des modes constitue en ce sens une véritable subversion, puisqu'elle remet en cause les fondements sur lesquels toute la poétique classique et romantique reposait. Ce faisant, Bertrand pose un geste digne du génie romantique qui répond de manière exemplaire aux revendications génériques d'un Stendhal (dans Racine et Shakespeare) ou d'un Hugo (dans la préfacede Cromwell). Surtout, Bertrand indique là le caractère actif du processus de transposition, lequel demande une réflexion d'ordre générique sur la littérature et ses possibilités. La transposition, essentiellement une pratique de transplantation, procède largement de l'ordre scripturaire. Elle signale la dimension auctoriale du genre.

Toute combinaison générique résulte d'un choix auctorial. À cet égard, la transposition semble la plus active de tous les processus de transformations génériques, puisqu'elle découle essentiellement d'une stratégie de la part de l'auteur, et dont le symptôme le plus visible sera un programme générique textuel bien précis ${ }^{19}$. De fait, le changement historique des genres provient de l'impulsion d'écrivains qui n'hésitent pas à repousser les cadres formels, thématiques ou modaux d'un genre donné. En témoigne de façon exemplaire la fameuse "querelle du Cid», précisément causée par le refus des poéticiens classiques d'admettre la transgression opérée par Corneille.

Ce qui ne revient pas à dire que le processus de transposition résulte toujours d'un choix conscient:

With kind and subgenre alike, the phase of assembly may of course be largely unconscious. The author perhaps thinks only of writing in a fresh way. It will often be his successors who first see the potential for genre and recognize, retrospectively, that assembly of a new form has taken place. (Fowler, 1982: 159)

Cela semble être le cas d'Aloysius Bertrand, qui utilise intentionnellement la transposition générique et modale sans arriver à nommer sa création, ce «nouveau genre de prose» (2000: 900) dont il a tant rêvé. Cette prose nouvelle pavera bien sûr la voie aux inventions poétiques de la modernité (Baudelaire, Mallarmé, Jacob). C'est toutefois la postérité qui y 
verra des poèmes en prose, et peut-être même les premiers poèmes en prose de la littérature française 20 .

De toute évidence, l'écrivain qui choisit de transposer dans un genre des procédés qui appartiennent originellement à un autre se pose d'ores et déjà en position de lecteur, puisqu'il cherche dans les œuvres qui le précèdent des modèles à retranscrire:

L'impulsion à mettre en forme se renouvelle sans cesse, et se vit toujours comme un acte originaire et unique; mais elle trouve dans la tradition littéraire des ouvrages formels qui ont été créés et développés par une impulsion à la mise en forme, parente de la précédente, et qui guidait d'autres artistes aux prises avec des problèmes analogues dans d'autres situations historiques.

(Viëtor, 1977: 492)

Cette posture particulière n'empêche toutefois pas le comportement dynamique de l'écrivain qui, agissant au sein du champ littéraire de son époque, peut tout de même influencer le développement des genres. "Genre also offers a challenge by provoking a free spirit to transcend the limitations of previous examples» (Fowler, 1982: 31). Baudelaire et Ravel, tous deux lecteurs de Bertrand, ont activement accompli de telles transpositions.

Dans une lettre à Arsène Houssaye aujourd'hui consacrée comme préface aux Petits poèmes en prose, Baudelaire confie avoir trouvé chez Bertrand l'inspiration nécessaire à une transcription poétique de la prose. Il écrit:

J'ai une petite confession à vous faire. C'est en feuilletant, pour la vingtième fois au moins, le fameux Gaspard de la Nuit, d'Aloysius Bertrand [...] que l'idée m'est venue de tenter quelque chose d'analogue, et d'appliquer à la description de la vie moderne, ou plutôt d'une vie moderne et plus abstraite, le procédé qu'il avait appliqué à la peinture de la vie ancienne, si étrangement pittoresque.

(1973: 21)

Lisant Bertrand, Baudelaire cherche à créer une "prose poétique, musicale sans rythme et sans rime» (1973 : 22) qui s'adaptera à la rêverie et aux mouvements de l'âme. Le poète des Fleurs du Mal produira finalement des poèmes en prose qui divergeront tout autant du programme qu'il se donne que des poèmes bertrandiens eux-mêmes, avec lesquels, par exemple, ils ne partagent pas leur structure cadrée. On pourrait dresser une assez longue liste des différences qui existent entre les poèmes de Bertrand et ceux de Baudelaire, mais il importe surtout de remarquer que le second agit volontairement: son inspiration n'est pas qu'imitative, elle est résolument engagée et vise une véritable rénovation des techniques et des visées des textes de Bertrand.

Ravel, pour sa part, s'inspire de trois poèmes de Gaspard de la Nuit, qu'il transpose en autant de pièces musicales. Le compositeur partage avec Bertrand un refus certain du développement et de l'explication, mais aussi de l'émotion, faisant dire à Cécile Raynaud: «Beaucoup d'éléments semblent donc rapprocher Bertrand et Ravel: les recherches formelles et l'ironie dans l'attitude créatrice» (1993: 164). Mais encore, Ravel arrive

[...] à l'idée que la musique n'exprime plus de façon latente certains états d'âmes, mais qu'elle parvient à signifier ce que dit un texte qui lui est adjoint [...] les trois «Poèmes» de Gaspard de la Nuit suivent un mouvement dramatique, ou des descriptions programmées par le texte. (ibid.: 169-170)

Adoptant les prescriptions textuelles du recueil dont il s'inspire, Ravel adapte un genre qu'il (re-)transpose dans le domaine musical, montrant bien qu' «un vrai créateur ne peut toucher à l'œuvre d'un autre sans y imprimer sa marque. La continuation devient ainsi, dans les meilleurs cas, prétexte à réécriture oblique» (Genette, 1982: 223).

Voilà comment Baudelaire et Ravel, lecteurs de Bertrand, se servent de la transposition générique pour devenir des créateurs à part entière plutôt que de simples imitateurs. Alors que l'imitation relève de la servilité et n'implique donc pas de choix auctoriaux, la transposition possède un caractère actif et volontaire, mais aussi certainement distinctif. Le traitement particulier, dans le domaine de la prose, des éléments formels et modaux qui appartiennent 
traditionnellement à d'autres arts permet justement à Bertrand de "passer à l'histoire» et d'acquérir $a$ posteriori le capital symbolique dont il aurait voulu jouir au cours de sa vie. La fortune de l'écrivain, pourtant au départ un provincial sans notoriété, atteste la vitalité propre à l'opération de transposition (générique, modale). En tant qu'opération de transplantation générique et modale, elle exige la volonté de l'écrivain qui, par son geste, signale la différence générique de sa production.

\section{N O TES}

1. B. Johnson écrit: «Impuissance, monstre, bâtard, hybride: la carte d'identité du poème en prose se lit comme un catalogue de troubles de la génération" (1976: 451).

2. Les auteurs ajoutent: «Plus précisément, [la transposition] désigne soit un changement de registre (du savant au ludique, du comique au tragique, etc.), soit un changement de domaine de validité (des concepts de la théorie littéraire en passant à la fiction [...]), soit encore une permutation d'éléments prototypiques qui produit un effet d'étrangeté à l'intérieur d'un genre, une sensation de "bougé", de semblable et d'autre à la fois; elle produit en somme un décalage formel potentiellement critique» (Dion, Fortier et Haghebaert, 2001 : 354).

3. La différenciation implique l'idée de dérivation: «cette différenciation peut être effectuée au moyen d'un retour à l'origine ou à la définition stricte du genre, d'une accentuation d'un trait générique particulier (accentuation éventuellement parodique) ou, au contraire, de la négation d'un élément définitionnel du genre " (Dion, Fortier et Haghebaert, 2001 : 353). L'hybridation se définit comme «la combinaison de plusieurs traits génériques hétérogènes [...], hiérarchisés ou non, en un même texte» (353).

4. Le texte raconte l'histoire d'un maître de chapelle qui, lors du bris d'une corde de sa viole, voit apparaître devant lui des personnages de la commedia dell'arte. La narrativité de «La viole de Gamba» et de «La poterne du Louvre» a fait l'objet d'un long développement dans Bonenfant (2002: 17-44).

5. Procédé auquel Bertrand attachait assez d'importance. Toujours dans ses directives typographiques, il écrit: «M. le metteur en pages blanchira les pièces comme il le jugera le plus convenable d'après les indications du manuscrit, mais toujours de manière à étendre et à faire foisonner la matière" (2000: 373).

6. On entendra le mot "génie» au sens qu'il prend à l'époque romantique.

7. Car l'époque romantique procède bel et bien au classement générique. Aucun auteur n'échappe alors vraiment au jugement classificatoire de la hiérarchie des genres. Sur cette question, voir Lambert (1985).

8. Ce qui n'est guère surprenant dans le contexte de l'époque. Pour les romantiques français, la poésie dépasse la question de l'écriture pour atteindre la sphère de l'ontologie. Par-delà le poème, elle constitue un langage que l'on trouve partout. Ainsi, Hugo écrit qu' «il y a du drame dans la poésie, et [...] de la poésie dans le drame» (1964: 1017), puisque tous deux participent d'une même expérience: l'expérience humaine.

9. Schaeffer (1989) a montré que le genre ne dépend pas seulement de l'aspect formel de l'œuvre. Qu'on pense au seul aspect pragmatique pour s'en convaincre.

10. «Le poème, le récit, la nouvelle, sont des formes compositionnelles pures [alors que] les formes architectoniques sont les formes que prennent les valeurs morales et physiques de l'homme esthétique, les formes de la nature perçues comme son environnement [...] ce sont les formes de la vie esthétique dans sa singularité» (Bakhtine, 1978: 35 36).

11. Plusieurs des poèmes en prose bertrandiens prennent la forme de la saynète, convoquant en conséquence le mode dramatique.

12. L'hypotaxe du récit bref (nouvelle, conte, chronique) permet de le distinguer du récit du poème en prose, généralement parataxique. 13. «Deux voyageurs se rencontrèrent le soir dans un étroit sentier » (2000: 364).

14. Le lecteur comprend rapidement que l'un est un honnête homme et l'autre, un brigand: «On devinait aisément que c'était un clerc du gai savoir [...] C'était Roland ou Don Quixote, ou tout autre chevalier célèbre» (2000: 364).

15. Cette identité, le sujet lyrique la cherche dans une union, voire une fusion, avec l'objet de son discours. S'il parle à titre individuel, le sujet lyrique parle à travers l'objet dont il parle. Qu'il s'agisse du lac de Lamartine, du pélican de Musset ou, plus généralement, de la nature hugolienne, l'énoncé lyrique, en tant qu'énoncé circonstanciel (Rabaté, 1996), s'identifie à son objet pour ne faire qu'un: «son objet n'est pas le but, mais l'occasion " (Hamburger, 1986: 234). À l'opposé, le sujet énonciatif de la prose lyrique ne s'identifie pas à son objet. «Il raconte un vécu personnel, mais sans la tendance à le présenter comme une vérité seulement subjective, comme son champ d'expérience, au sens prégnant de ce terme: il est, comme tout Je historique, orienté vers la vérité objective du narré » (ibid. : 275). C'est donc là qu'il faut faire la différence entre énonciation lyrique (du poème) et énonciation à la première personne (de la prose lyrique).

16. Elles sont: "Le clair de lune", «Les lavandières» et "La gourde et le flageolet" (Le Provincial, 47, vendredi 12 septembre 1828, p. 212).

17. Soulignons que ces procédés modaux de signification poétique, Bertrand les emprunte peut-être aussi aux genres narratifs brefs, et plus particulièrement au conte, genre dont on a souvent souligné le caractère énigmatique.

18. Sur le rapport entre la musique et la littérature dans l'œuvre d'Hoffmann, voir Giraud (1987).

19. Dans une perspective différente de la nôtre, Schaeffer parle à cet égard de «régime auctorial» des textes. Il écrit: «En effet, [...] au niveau de la création des œuvres les phénomènes génériques correspondent pour l'essentiel, comme il semble raisonnable de l'admettre, à des faits intentionnels de choix, d'imitation et de transformation (quelles que soient les motivations de ces décisions [...]) » (Schaeffer, 1989: 148). 20. «Il est rare d'assister à la naissance d'un genre littéraire. Plus rare encore de pouvoir la rattacher au nom d'un écrivain particulier. Cette conjonction s'est pourtant opérée dans le cas d'Aloysius Bertrand, inventeur incontestable du poème en prose français» (Milner, 1980: 7). 


\section{RÉFÉRENCES BIBLIO G RAPH IQ U ES}

BAKHTINE, M. [1978] : «Le problème du contenu, du matériau et de la forme dans l'œuvre littéraire", Esthétique et théorie du roman, trad. de D. Olivier, Paris, Gallimard, coll. "Tel», 21-82.

Baudelaire, C. [1973]: Petits poèmes en prose, (sous la dir. de R. Kopp), Paris, Gallimard.

Bernard, S. [1959]: Le Poème en prose de Baudelaire jusqu'à nos jours, Paris, Nizet.

Bertrand, A. [2000]: CEuvres complètes, (sous la dir. de H. Hart Poggenburg), Paris, Champion, coll. «Textes de littérature moderne et contemporaine $\mathrm{n}^{\circ} 32 \%$.

BONENFANT, L. [2002] : Les Avatars romantiques du genre. Transferts génériques dans l'œuvre d'Aloysius Bertrand, Québec, Nota bene, coll. "Littérature(s)».

CHORÉ, P. [1993]: "À propos de Bertrand et de Callot», dans F. Claudon (dir.), Les Diableries de la Nuit. Hommage à Aloysius Bertrand, Dijon, EUDijon, coll. "Figures libres", 95-109.

Claudon, F. [1988]: "Gaspard de la Nuit et le pictural», Gazette des Beaux-Arts, VI e période, tome CXI, 1428 -1429 e livraisons, 77-80.

CORBAT, H. [1975]: Hantise et imagination chez Aloysius Bertrand, Paris, José Corti.

De SERMET, J. [1996]: «L'adresse lyrique», dans D. Rabaté (dir.), Figures du sujet lyrique, Paris, P.U.F., coll. "Perspectives littéraires", 81-97. Dion, R., F. FORTIER et É. HAGHEBAERT 2001] : "La dynamique des genres, suite. Conclusion", dans R. Dion, F. Fortier et É. Haghebaert (sous la dir.), Enjeux des genres dans les écritures contemporaines, Québec, Nota bene, coll. "Cahiers du CRELIQ", 351-362.

FOwLER, A. [1974]: "The Life and Death of Literary Forms ", dans R. Cohen (dir.), New Directions in Literary History, London, Routledge \& Kegan Paul, 77-94;

[1982]: Kinds of Literature: An Introduction to Literary Kinds, Cambridge, Harvard University Press.

Genette, G. [1982]: Palimpsestes, Paris, Seuil, coll. «Poétique».

GIRAUD, J. [1987]: «Éléments musicaux dans l'œuvre littéraire d'E. T.

A. Hoffmann", dans A. Montandon (dir.), E. T. A. Hoffmann et la musique: actes du colloque international de Clermont-Ferrand, Berne, Peter Lang, 207-238.

Hamburger, K. [1986] : Logique des genres littéraires, trad. de P. Cadiot, Paris, Seuil, coll. "Poétique».

Hugo, V. [1964]: Euvres poétiques I, (sous la dir. de P. Albouy), Paris, Gallimard, coll. "Bibliothèque de la Pléiade».

JoHnson, B. [1976]: «Quelques conséquences de la différence anatomique des textes. Pour une théorie du poème en prose", Poétique, $\mathrm{n}^{\circ} 28,450-465$.

LAMBERT, J. [1985]: «Vers et prose à l'époque romantique, ou la hiérarchie des genres dans les lettres françaises ", dans Du romantisme au Surnaturalisme. Hommage à Claude Pichois, Neuchâtel, La Baconnière, coll. "Langages ", 39-55.

Milner, M. [1980]: "Préface», Gaspard de la Nuit. Fantaisies à la manière de Rembrandt et de Callot, Paris, Gallimard, 7-57.

RABATÉ, D. [1996] : «Énonciation poétique, énonciation lyrique», dans D. Rabaté (dir.), Figures du sujet lyrique, Paris, P.U.F., coll. "Perspectives littéraires", 65-79.

RAYNAUD, C. [1993]: "Gaspard de la Nuit: Fantaisies à la manière de Ravel», dans F. Claudon (dir.), Les Diableries de la Nuit. Hommage à Aloysius Bertrand, Dijon, EUDijon, coll. «Figures libres », 161-185. SANDRAS, M. [1995]: Lire le poème en prose, Paris, Dunod. SCHAEFFER, J.-M. [1989]: Qu'est-ce qu'un genre littéraire?, Paris, Seuil, coll. "Poétique».

Schleuning, P. [1971]: The Fantasia I, trad. de A. C. Howie, Cologne, Arno Volk Verlag.

SCOTT, D. [1989] : Pictorialist Poetics, Cambridge, Cambridge University Press.

VANHĖSE CiCCHETti, G. [1979]: «L'archaïsme stylistique dans Gaspard de la Nuit", Micromégas. Rivista di Studi e Confronti Italiani e Francesi, 6: $1,1-25$.

VIËTOR, K. [1977]: «L'histoire des genres littéraires ", Poétique, 32, 490506.

Vincent-Munnia, N. [1996]: Les Premiers Poèmes en prose: généalogie d'un genre dans la première moitié du dix-neuvième siècle français, Paris, Champion, coll. "Romantisme et modernités I». 